Kuperus - die Existenz von zwei schon früher durch TANGEN ${ }^{12}$ beobachteten Resonanzen bestätigt werden; drei weitere wurden erstmalig gefunden. Die Resonanzen konnten durch Verwendung von Auffängern aus $\mathrm{AgCl}$, in denen die Chlorisotope zu $95,2 \%\left({ }^{35} \mathrm{Cl}\right)$ bzw. $60,5 \%\left({ }^{37} \mathrm{Cl}\right)$ angereichert waren ${ }^{5}$, eindeutig dem jeweiligen Isotop zugeordnet werden (s. Abb. 1).

Die in Tab. 1 aufgeführten experimentellen Halbwertsbreiten, die, wie in II dargelegt, aus dem An- stieg der Ausbeutekurve an der Resonanz gewonnen wurden, stellen lediglich obere Grenzen für die natürlichen Gesamt-Resonanzhalbwertsbreiten dar. Diese lassen sich mit Hilfe einer ebenfalls in II näher erläuterten theoretischen Betrachtung für die angegebenen Resonanzen zu kleiner als $\mathrm{l} \mathrm{eV}$ abschätzen.

Herrn Prof. Dr. H. FräNZ danken wir für förderndes Interesse, Herrn Ing. H.-J. Barrenscheen für wertvolle Mitarbeit bei den Messungen.

\title{
Zur Elektronenoptik elektrostatischer Beschleuniger im relativistischen Bereich
}

\author{
Von Joachim Kessler \\ Aus dem Physikalischen Institut der Technischen Hochschule Karlsruhe \\ (Z. Naturforschg. 16 a, 1138-1146 [1961] ; eingegangen am 22. Juli 1961)
}

\begin{abstract}
The properties of electrostatic lenses for relativistic particles are described by means of transfer matrices. Using GANs' polygon-method, the matrices are calculated by integrating the equation of motion of relativistic particles in electric fields that have rotational symmetry. These general results are used for calculating the first-order approximations of the characteristic data for two-cylinder lenses. Numerical evaluation shows that the lens properties at a fixed $V_{2} / V_{1}$ vary only slowly with increasing energy ( $V_{1}$ and $V_{2}$ being the potentials of the lens). The results are compared with those calculated by Timm for the nonrelativistic case, which can be seen in our results as a special case. The easiest way of applying the derived formulas to the case of multistage accelerator tubes is discussed.
\end{abstract}

Für die Berechnung elektrostatischer Beschleuniger war die Arbeit von Timm ${ }^{1}$ ein wesentlicher Fortschritt. Die Abbildungseigenschaften rotationssymmetrischer und ebener Felder wurden dort durch Angabe der „Abbildungsmatrizen“ vollständig beschrieben. Ein großer Vorteil dieser Methode besteht darin, daß die Berechnung mehrerer hintereinandergeschalteter Linsen, wie sie in Beschleunigungsrohren benutzt werden, sehr einfach wird. Man braucht hier nur die entsprechenden Abbildungsmatrizen miteinander zu multiplizieren, um die Abbildungsmatrix des Gesamtsystems zu erhalten.

Der Timmschen Arbeit lag das Problem zugrunde, die Abbildungseigenschaften eines Ionenbeschleunigungsrohres zu ermitteln, das in Verbindung mit einem VAN DE GRAAFF-Generator betrieben wurde. Deshalb interessierten Einflüsse der relativistischen Massenveränderlichkeit dort nicht. Beim Betrieb

1 U. Tiмм, Z. Naturforschg. 10 a, 593 [1955].

2a W. W. Hansen u. D. L. Webster, Rev. Sci. Instrum. 7, 17 [1936]. eines gleichartigen Beschleunigers für Elektronen befindet man sich dagegen praktisch immer im relativistischen Bereich, so daß sich die Trmmschen Ergebnisse nicht ohne weiteres übernehmen lassen.

Deshalb sollen hier auf der Grundlage der Matrizendarstellung die Abbildungseigenschaften elektrostatischer Rohrlinsen für relativistische Teilchen berechnet werden. Das Ziel ist es, auch im relativistischen Bereich geschlossene Formeln anzugeben, aus denen sich die Linsendaten bei beliebigen Potentialverhältnissen an der Linse und beliebigen Teilchenenergien ablesen lassen.

Bisher wurden im relativistischen Gebiet nur in Spezialfällen Linsendaten angegeben, z. B. von HaNSEN und Webster ${ }^{2 a}$ für den Fall sehr schwacher Linsen oder von $\mathrm{BAS}^{2 \mathrm{~b}}$ u. a., die einen speziellen Beschleuniger mit einer elektronischen Rechenmaschine sehr genau berechneten.

2b E. B. Bas, L. Preuss u. W. Schneider, Z. angew. Math. Phys. 10, 533 [1959]. 


\section{Zusammenhang der charakteristischen Linsendaten mit der Abbildungsmatrix}

Wir stellen in diesem Abschnitt die von Timm ${ }^{1}$ gewonnenen Ergebnisse über den allgemeinen $\mathrm{Zu}$ sammenhang der charakteristischen Linsendaten mit den Elementen der Abbildungsmatrix zusammen und halten uns dabei an die von ihm eingeführten Bezeichnungen.

Die Abbildungseigenschaften einer Linse werden vollständig beschrieben durch Angabe der bild- und dingseitigen Brennweiten $f$ und Brennpunktslagen $F$. Die bekannte Definition dieser Größen wird in den ersten beiden Abbildungen veranschaulicht. Die Größen $F$ oder $f$ werden häufig ersetzt durch die Abstände $\Delta F$ der Hauptebenen $\mathrm{H}$ von den zugehörigen Linsenrändern. Es gilt

$$
f=F-\Delta F
$$

(man beachte Vorzeichen und Nullpunkte der Zählung).

Die Abb. 1 und 2 stellen übrigens die Daten einer beschleunigenden Rohrlinse, wie sie die Timsschen Formeln für den Fall $V_{2} / V_{1}=4$ liefern, maßstäblich dar (e $V_{1}$ bzw. $e V_{2}$ sind Eintritts- bzw. Austrittsenergie der beschleunigten Teilchen, $e=$ Elementarladung).

Wir wollen jetzt die Abbildungsmatrix einführen. Ein Elektronenstrahl trete im Abstand $r_{\mathrm{e}}$ von der Achse mit der Neigung $r_{\mathrm{e}}{ }^{\prime}=(\mathrm{d} r / \mathrm{d} z)_{\mathrm{e}}$ in das Linsenfeld ein und komme im Abstand $r_{\mathrm{a}}$ mit der Neigung $r_{\mathrm{a}}{ }^{\prime}$ wieder heraus (Abb. 3). Man überlegt sich leicht, daß sich die Daten $r_{\mathrm{a}}$ und $r_{\mathrm{a}}{ }^{\prime}$ des austretenden Strahls aus denen des einfallenden Strahls in der Form

$$
\begin{aligned}
& r_{\mathrm{a}}=a_{11} r_{\mathrm{e}}+a_{12} r_{\mathrm{e}}{ }^{\prime}, \\
& r_{\mathrm{a}}{ }^{\prime}=a_{21} r_{\mathrm{e}}+a_{22} r_{\mathrm{e}}{ }^{\prime}
\end{aligned}
$$

linear kombinieren lassen, sofern die Bahn innerhalb der Linse durch eine lineare, homogene, gewöhnliche Diff.-Gl. 2. Ordnung beschrieben wird. Oder in Matrizenschreibweise

$$
\mathfrak{r}_{\mathrm{a}}=\mathfrak{U} \mathfrak{r}_{\mathrm{e}} \quad \text { mit } \quad \mathfrak{U}=\left(\begin{array}{ll}
a_{11} & a_{12} \\
a_{21} & a_{22}
\end{array}\right) \quad \text { und } \quad \mathfrak{r}=\left(\begin{array}{c}
r \\
r^{\prime}
\end{array}\right) \text {. }
$$

$\mathfrak{A}$ ist die Abbildungsmatrix.

Mit Hilfe einfacher geometrischer Betrachtungen überlegt man sich an Hand der Abb. 1 und 2 leicht (vgl. Trмm ${ }^{1}$ ), daß zwischen den Linsendaten und den Elementen der Abbildungsmatrix folgender $\mathrm{Zu}$ sammenhang besteht:

$$
\begin{array}{lll}
F_{\mathrm{a}}=-\frac{a_{11}}{a_{21}}, & \Delta F_{\mathrm{a}}=\frac{1-a_{11}}{a_{21}}, \quad f_{\mathrm{a}}=-\frac{1}{a_{21}}, \\
F_{\mathrm{e}}=-\frac{a_{22}}{a_{21}}, & \Delta F_{\mathrm{e}}=\frac{D-a_{22}}{a_{21}}, \quad f_{\mathrm{e}}=-\frac{D}{a_{21}},
\end{array}
$$

wobei $D=$ Det. $\mathfrak{U}=a_{11} a_{22}-a_{21} a_{12}$.

Drückt man umgekehrt die Matrixelemente durch die Linsendaten aus, so folgt

$$
\mathfrak{U}=\frac{\mathbf{l}}{f_{\mathrm{a}}}\left(\begin{array}{cc}
\boldsymbol{F}_{\mathrm{a}} & f_{\mathrm{a}} f_{\mathrm{e}}-F_{\mathrm{a}} F_{\mathrm{e}} \\
-1 & F_{\mathrm{e}}
\end{array}\right) .
$$

Man erkennt hieraus, daß das Problem der Bestimmung der Linsendaten auf die Bestimmung der $\mathrm{Ab}$ bildungsmatrix zurückgeführt ist. Daraus lassen sich dann alle interessierenden Abbildungseigenschaften bestimmen, wie etwa Abbildungsgleichung, Lateralund Angularvergrößerung.

\section{Allgemeine Berechnung der Abbildungsmatrix nach der Polygonmethode}

Wir wollen jetzt die Abbildungsmatrix für die Bewegung relativistischer Teilchen in elektrostati-

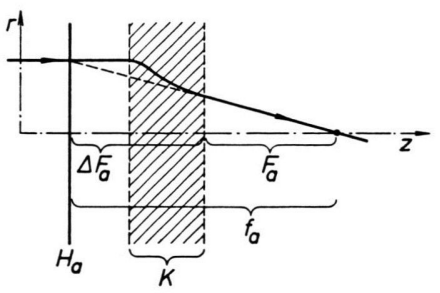

Abb. 1. Zur Definition der bildseitigen Linsendaten. Alle Größen werden in der Einfallsrichtung positiv gezählt. Die Zählung von $F_{\mathrm{a}}$ und $\Delta F_{\mathrm{a}}$ beginnt am bildseitigen Linsenrand, die von $f_{\mathrm{a}}$ an der Hauptebene $\mathrm{H}_{\mathrm{a}}$. $K$ ist die effektive Länge der Linse.

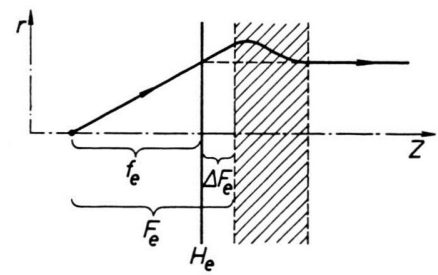

Abb. 2. Zur Definition der dingseitigen Linsendaten. Alle Größen werden in der Einfallsrichtung negativ gezählt. Die Zählung von $F_{\mathrm{e}}$ und $\Delta F_{\mathrm{e}}$ beginnt am dingseitigen Linsenrand, die von $f_{\mathrm{e}}$ an der Hauptebene $\mathrm{H}_{\mathrm{e}}$.

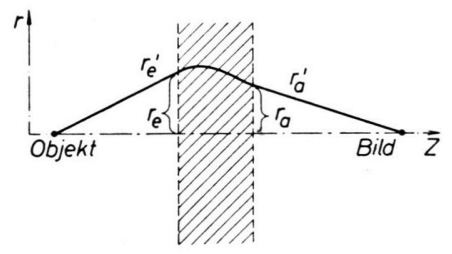

Abb. 3. Zur Definition der Daten des Strahls. 
schen Rohrlinsen berechnen. Wenn wir uns auf den Bereich der Gaussschen Dioptrik (flache, achsennahe Strahlen) beschränken, lautet die Bewegungsgleichung für relativistische Teilchen in rotationssymmetrischen elektrostatischen Feldern ${ }^{3}$

$$
\begin{array}{r}
\sqrt{(1+\varepsilon \Phi) \Phi \frac{\mathrm{d}}{\mathrm{d} z}\left[\sqrt{(1+\varepsilon \Phi) \Phi} \frac{\mathrm{d} r}{\mathrm{~d} z}\right]} \\
+\frac{1}{4}(1+2 \varepsilon \Phi) \Phi^{\prime \prime} r=0,
\end{array}
$$

oder umgeformt

$$
r^{\prime \prime}=-\frac{1+2 \varepsilon \Phi}{2(1+\varepsilon \Phi) \Phi}\left[\Phi^{\prime} r^{\prime}+\frac{1}{2} \Phi^{\prime \prime} r\right] .
$$

Dabei ist $\Phi$ das Potential längs der Achse, dessen additive Konstante so gewählt ist, daß $\Phi$ dort verschwindet, wo die Teilchengeschwindigkeit Null ist.

$$
\varepsilon=e / 2 m_{0} c^{2}
$$

bestimmt die relativistische Korrektur. $m_{0}$ und $e$ sind Ruhmasse und Betrag der Ladung des beschleunigten Teilchens ( $\Phi$ wird also für positive und negative Teilchen positiv gerechnet).

Für Elektronen wird

für Protonen

$$
\varepsilon=0,978 \cdot 10^{-6}\left[\mathrm{~V}^{-1}\right],
$$

$$
\varepsilon=0,539 \cdot 10^{-9}\left[\mathrm{~V}^{-1}\right] .
$$

Bei vorgegebener Linse, wenn also $\Phi$ und damit auch seine Ableitungen längs der Achse bekannt sind, läßt sich die Bahnkurve $r(z)$ aus (7) berechnen. Diese Gl. läßt sich aber selbst im nichtrelastivistischen Fall $(\varepsilon=0)$ für beliebiges Achsenpotential $\Phi$ nicht allgemein integrieren. GANS ${ }^{4}$ approximierte deshalb das Achsenpotential durch einen Polygonzug (vgl. Abb. 4) und führte die Integration der nichtrelativistischen Gleichung schrittweise durch. Seine Ergebnisse bilden die Grundlage der Timmschen Matrizendarstellung.

Wir wollen jetzt nach der Gansschen Polygonmethode, bei der man durch genügend feine Unterteilung der Strecke $K$ jede gewünschte Genauigkeit erreichen kann, die relativistische $\mathrm{Gl}$. (7) integrieren. Dabei kommen nur 2 verschiedenartige Schritte vor: Integration über einen Abschnitt und Integration über eine Ecke. Wir führen diese Schritte nacheinander durch.

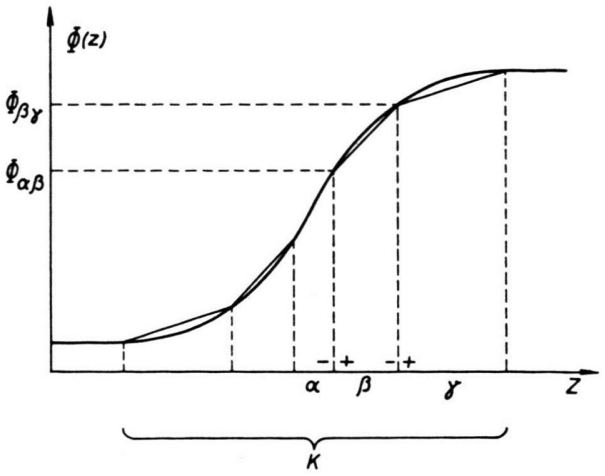

Abb. 4. Approximation des Achsenpotentials durch Polygonzug.

a) Integration über einen Abschnitt $\beta$ :

Wegen $\Phi^{\prime \prime}=0$ wird aus (7)

$$
r^{\prime \prime}=-\frac{1+2 \varepsilon \Phi}{2(1+\varepsilon \Phi) \Phi} \Phi^{\prime} r^{\prime}
$$

oder

$$
\frac{\mathrm{d}}{\mathrm{d} z} \ln r^{\prime}=-\frac{1}{2} \frac{\mathrm{d}}{\mathrm{d} z} \ln (1+\varepsilon \Phi) \Phi .
$$

Daraus folgt

$$
r^{\prime}(z)=\sqrt{\frac{\left(1+\varepsilon \Phi_{\alpha \beta}\right) \Phi_{\alpha \beta}}{[1+\varepsilon \Phi(z)] \Phi(z)}} \cdot r_{\alpha \beta+}^{\prime}
$$

für $z_{\alpha \beta+} \leq z \leq z_{\beta_{\gamma}-}$.

Dabei kennzeichnet der Index $\alpha \beta^{+}$bzw. $\alpha \beta^{-}$den rechts- bzw. linksseitigen Grenzwert an der Ecke $\alpha \beta$.

Integration von (9) zwischen den Grenzen $z_{\alpha \beta}+$ und $z_{\beta \gamma}$ - liefert

$$
\begin{array}{r}
r_{\beta \gamma}=r_{\alpha \beta}++\frac{\sqrt{\left(1+\varepsilon \Phi_{\alpha \beta}\right) \Phi_{\alpha \beta}}}{\Phi_{\beta}^{\prime} \sqrt{\varepsilon}} r_{\alpha \beta+}^{\prime} \quad(10 \mathrm{a}) \\
\cdot \ln \frac{1+2 \varepsilon \Phi_{\beta \gamma}+2 \sqrt{\varepsilon} \Phi_{\beta \gamma}\left(\varepsilon \Phi_{\beta \gamma}+1\right)}{1+2 \varepsilon \Phi_{\alpha \beta}+2 \sqrt{\varepsilon \Phi_{\alpha \beta}\left(\varepsilon \Phi_{\alpha \beta}+1\right)}} .
\end{array}
$$

Wenn man noch berücksichtigt, daß aus (9) folgt

$$
r_{\beta \gamma^{-}}^{\prime}=\sqrt{\frac{\left(1+\varepsilon \Phi_{\alpha \beta}\right) \Phi_{\alpha \beta}}{\left(1+\varepsilon \Phi_{\beta \gamma}\right) \Phi_{\beta \gamma}}} \cdot r_{\alpha \beta+}^{\prime},
$$

kann man (10 a) und (10 b) in Matrizenform schreiben:

$$
\ddot{r}_{\beta \gamma-}=\left(\begin{array}{c}
1 \frac{\sqrt{\left(1+\varepsilon \Phi_{\alpha \beta}\right) \Phi_{\alpha \beta}}}{\Phi_{\beta}^{\prime} V \bar{\varepsilon}} \ln \frac{1+2 \varepsilon \Phi_{\beta \gamma}+2 \sqrt{\varepsilon \Phi_{\beta \gamma}\left(\varepsilon \Phi_{\beta \gamma}+1\right)}}{1+2 \varepsilon \Phi_{\alpha \beta}+2 \sqrt{\varepsilon \Phi_{\alpha \beta}\left(\varepsilon \Phi_{\alpha \beta}+1\right)}} \\
0 \sqrt{\frac{\left(1+\varepsilon \Phi_{\alpha \beta}\right) \Phi_{\alpha \beta}}{\left(1+\varepsilon \Phi_{\beta \gamma}\right) \Phi_{\beta \gamma}}}
\end{array}\right) \mathfrak{r}_{\alpha \beta+}
$$

3 W. Glaser, Grundlagen der Elektronenoptik, Springer-Ver- ${ }^{4}$ R. Gavs, Z. techn. Phys. 18, 41 [1937]. lag, Wien 1952, S. 144. 
Damit haben wir für den Abschnitt $\beta$ Austrittsort und Austrittstangente durch die entsprechenden Eintrittsdaten in der gewünschten Form (3) dargestellt.

In Beschleunigern kommt häufig der Fall vor, daß $\Phi_{\beta}^{\prime}=0$ wird, weil zwischen den Beschleunigungsstrecken im allgemeinen feldfreie Gebiete liegen. Die Beziehung (10) gilt auch in diesem Grenzfall noch. Sie nimmt dann die einfache Form

$$
\mathfrak{r}_{\beta \gamma-}=\left(\begin{array}{cc}
1 & K_{\beta} \\
0 & 1
\end{array}\right) \mathfrak{r}_{\alpha \beta+}
$$

an, was man sich leicht überlegt, wenn man beachtet, da $\beta$ in diesem Fall $\Phi_{\beta \gamma}$ gegen $\Phi_{\alpha \beta}$ geht und daß $\ln (1+x) \rightarrow x$ für kleine $x$ gilt. $K_{\beta}$ ist dabei die Länge des feldfreien Abschnitts. Die Gültigkeit der Gl. (11) im feldfreien Raum kann man sich auch direkt überlegen.

b) Integration über eine Ecke $\beta \gamma$ :

Hier gilt $\Phi^{\prime \prime} \rightarrow \infty$ und $\Phi, \Phi^{\prime}, r, r^{\prime}$ endlich. Deshalb folgt aus (7)

$$
r^{\prime \prime} \rightarrow-\frac{1+2 \varepsilon \Phi}{4(1+\varepsilon \Phi) \Phi} \Phi^{\prime \prime} r .
$$

Außerdem gilt

$$
\frac{1}{4} \frac{(1+2 \varepsilon \Phi) \Phi^{\prime \prime} r}{(1+\varepsilon \Phi) \Phi}=\frac{\mathrm{d}}{\mathrm{d} z}\left(\frac{1}{4} \frac{(1+2 \varepsilon \Phi) \Phi^{\prime} r}{(1+\varepsilon \Phi) \Phi}\right),
$$

da nach der Differentiation alle Glieder auf der rechten Seite vernachlässigbar sind, die nicht den Faktor $\Phi^{\prime \prime}$ enthalten. Integration über die Ecke liefert also

$r_{\beta \gamma}^{\prime}=r_{\beta \gamma}^{\prime}$

$$
+\frac{1}{4} \frac{\left(1+2 \varepsilon \Phi_{\beta \gamma}\right)}{\left(1+\varepsilon \Phi_{\beta \gamma}\right) \Phi_{\beta \gamma}}\left(\Phi_{\beta \gamma}^{\prime}--\Phi_{\beta \gamma+}^{\prime}\right) r_{\beta \gamma-} .
$$

Berücksichtigt man, daß

$$
r_{\beta \gamma}+=r_{\beta \gamma},,
$$

so lassen sich die Werte von $r$ und $r^{\prime}$ hinter der Ecke durch die Werte vor der Ecke in Matrizenform ausdrücken:

$$
\mathfrak{r}_{\beta \gamma^{+}}=\left(\begin{array}{cc}
1 & 0 \\
\frac{\left(1+2 \varepsilon \Phi_{\beta \gamma^{\prime}}\right)\left(\Phi_{\beta \gamma^{\prime}}^{\prime}-\Phi_{\beta \gamma^{+}}^{\prime}\right)}{4\left(1+\varepsilon \Phi_{\beta \gamma}\right) \Phi_{\beta \gamma}} & 1
\end{array}\right) \mathfrak{r}_{\beta \gamma^{-}-} .
$$

Um die Bewegungsgleichung (7) über die ganze Länge $K$ der Linse zu integrieren, braucht man nur die beiden eben gemachten Schritte mehrmals nacheinander durchzuführen. Der große Vorteil der Matrizendarstellung ist, da $ß$ man dazu nur die jeweiligen Matrizen miteinander zu multiplizieren braucht; z. B. ergibt sich in dem einfachsten Fall, in dem

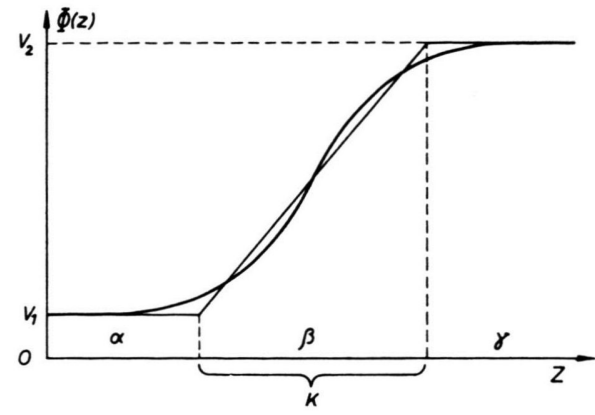

Abb. 5. Einfachste Approximation der Rohrlinse.

man das Achsenpotential durch eine Gerade approximiert (vgl. Abb. 5) :

$\mathfrak{r}_{\beta \gamma+}=\mathfrak{U}_{\beta \gamma} \cdot \mathfrak{r}_{\beta \gamma}-=\mathfrak{U}_{\beta \gamma} \cdot \mathfrak{U}_{\beta} \cdot \mathfrak{r}_{\alpha \beta+}=\mathfrak{U}_{\beta \gamma} \cdot \mathfrak{U}_{\beta} \cdot \mathfrak{U}_{\alpha \beta} \cdot \mathfrak{r}_{\alpha \beta-}$,

wobei die doppelt-indizierten Matrizen zu den Ecken gehören, also wie in (13) darstellbar sind, während die einfach indizierten Matrizen zu den Abschnitten gehören und damit eine Form wie in (10) haben.

\section{Die einfachste Approximation der Rohrlinse}

Wir wollen jetzt die allgemeinen Überlegungen des letzten Abschnitts auf den Fall anwenden, daß wir das Achsenpotential einer Rohrlinse in einfachster Weise, wie in Abb. 5, approximieren.

Wenn wir mit $V_{1}$ und $V_{2}$ die Potentiale der Rohrstücke bezeichnen (additive Konstante so gewählt, daß $V=0$, wenn Teilchengeschwindigkeit Null ist), dann wird

$$
\begin{gathered}
\Phi_{\alpha \beta}=V_{1}, \quad \Phi_{\beta \gamma}=V_{2}, \quad \Phi_{\alpha \beta-}^{\prime}=\Phi_{\beta \gamma^{+}}^{\prime}=0, \\
\Phi_{\alpha \beta+}^{\prime}=\Phi_{\beta}^{\prime}=\Phi_{\beta \gamma^{-}}^{\prime}=\left(V_{2}-V_{1}\right) / K .
\end{gathered}
$$

Mit diesen Bezeichnungen und $N=V_{2} / V_{1}$ erhalten wir bei der Integration über die rechte Ecke nach (13)

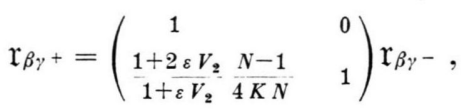

bei Integration über den mittleren Abschnitt nach (10)

$\mathfrak{r}_{\beta \gamma-}$

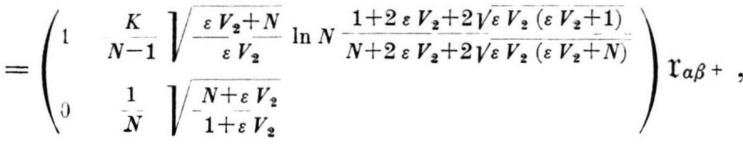

bei Integration über die linke Ecke nach (13)

$$
\mathfrak{r}_{\alpha \beta+}=\left(\begin{array}{cc}
1 & 0 \\
-\frac{\left(N+2 \varepsilon V_{2}\right)(N-1)}{4 K\left(N+\varepsilon V_{2}\right)} & 1
\end{array}\right) \mathfrak{r}_{\alpha \beta-} .
$$


Beachten wir, daß mit den Bezeichnungen des 1 . Abschnittes $\mathfrak{r}_{\beta \gamma}+=\mathfrak{r}_{\mathrm{a}}$ und $\mathfrak{r}_{\alpha \beta-}=\mathfrak{r}_{\mathrm{e}}$ ist, so folgt aus (15), (16) und (17), wenn wir die drei dort auftretenden Matrizen miteinander multiplizieren.

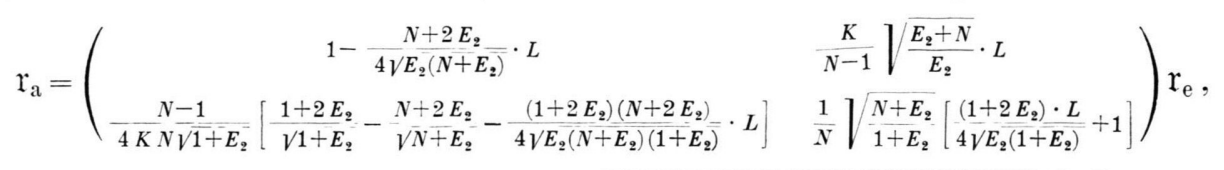

mit $\quad E_{2}=\varepsilon V_{2}$

und $L=\ln N \frac{1+2 E_{2}+2 \sqrt{E_{2}\left(1+E_{2}\right)}}{N+2 \mathrm{E}_{2}+2 \sqrt{E_{2}\left(N+E_{2}\right)}}$.

Damit haben wir Austrittsort und -tangente des Strahls mit Eintrittsort und -tangente in der Form (3) miteinander verknüpft und die Abbildungsmatrix $\mathfrak{U}$ gewonnen.

Ermittelt man daraus die Daten der Linse gemäß den Gln. (4), so folgt mit der Abkürzung

$$
\begin{aligned}
& v=(N-1)\left[4 \sqrt{E_{2}\left(N+E_{2}\right)}\left(1+2 E_{2}\right)\right. \\
& -4 \sqrt{E_{2}\left(1+E_{2}\right)}\left(N+2 E_{2}\right) \\
& \left.-\left(1+2 E_{2}\right)\left(N+2 E_{2}\right) L\right]: \\
& F_{\mathrm{a}} / K=-4 N\left(1+E_{2}\right)\left[4 \sqrt{E_{2}} \overline{\left(N+E_{2}\right)}\right. \\
& \left.-\left(N+2 E_{2}\right) L\right] / v, \\
& \Delta F_{\mathrm{a}} / K=4 N\left(1+E_{2}\right)\left(N+2 E_{2}\right) L / v, \\
& f_{\mathrm{a}} / K=-16 N\left(1+E_{2}\right) \sqrt{E_{2}\left(N+E_{2}\right)} / v, \\
& F_{\mathrm{c}} / K=-4\left(N+E_{2}\right)\left[4 V E_{2}\left(1+E_{2}\right)\right. \\
& \left.+\left(1+2 E_{2}\right) L\right] / v, \\
& \Delta F_{\mathrm{e}} / K=-4\left(N+E_{2}\right)\left(1+2 E_{2}\right) L / v, \\
& f_{\mathrm{e}} / K=-16\left(N+E_{2}\right) \sqrt{E_{2}\left(1+E_{2}\right)} / v .
\end{aligned}
$$

Diese Ausdrücke sind zwangsläufig etwas komplizierter als die entsprechenden nichtrelativistischen Formeln, da die Abbildungseigenschaften einer elektrostatischen Linse im relativistischen Fall nicht nur vom Potentialverhältnis $N$, sondern auch vom absoluten Wert des Potentials abhängen. Aus (20) läßt sich ablesen, daß zwischen den zusammengehörigen bild- und dingseitigen Linsendaten einfache Beziehungen bestehen, deren bekannteste die allgemeine Gleichung

$$
f_{\mathrm{e}}=(1 / N) \sqrt{\left(N+E_{2}\right) /\left(1+E_{2}\right)} f_{\mathrm{a}}
$$

ist.

Man überzeugt sich leicht davon, daß die Trmmschen Ergebnisse in den hier angegebenen Resultaten als Spezialfälle enthalten sind: Im Grenzfall $E_{2} \rightarrow 0$ (bzw. $\varepsilon \rightarrow 0$ im Abschnitt 2) gehen sämtliche Abbildungsmatrizen und Linsendaten in die entsprechenden nichtrelativistischen Ausdrücke über.
Durch die Gln. (20) sind die Abbildungseigenschaften der Linse vollständig beschrieben;

Abbildungsgleichung $\quad A^{\prime} E^{\prime}=f_{\mathrm{a}} \cdot f_{\mathrm{e}}$,

Lateralvergrößerung $\quad \beta=-A^{\prime} / f_{\mathrm{a}}$

und Angularvergrößerung (vgl. GLASER ${ }^{5}$ )

$$
\gamma=\frac{1}{\beta N} \sqrt{\frac{N+E_{2}}{1+E_{2}}}
$$

lassen sich mit ihrer Hilfe sofort hinschreiben. $E^{\prime}$ bzw. $A^{\prime}$ ist hierin der Abstand des Gegenstands bzw. Bildes vom dingseitigen bzw. bildseitigen Brennpunkt („Brennpunktsweite“).

Die Umkehrbarkeit des Strahlengangs sieht man den Gln. (20) sofort an. Ersetzt man nämlich in (20) $N=V_{2} / V_{1}=E_{2} / E_{1} \operatorname{durch} \mathrm{l} / N$ und $E_{2} \operatorname{durch} E_{1}=E_{2} / N$ (das bedeutet: Man betrachtet ein Teilchen, das in umgekehrter Richtung durch die Linse läuft), so gehen die bildseitigen in die dingseitigen Linsendaten über und umgekehrt.

Um einen Überblick über die Linseneigenschaften im relativistischen Bereich zu erhalten, wurden die Gln. (20) für 3 verschiedene Energien numerisch ausgewertet, und zwar für $E_{2}=0,1,1$ und 10, was wegen $E_{2}=e V_{2} / 2 m_{0} c^{2}$ Elektronenendenergien von $102,2 \mathrm{keV}, 1,022 \mathrm{MeV}$ und $10,22 \mathrm{MeV}$ entspricht. In Tab. 1 sind die Ergebnisse für verschiedene Werte des Potentialverhältnisses $N$ an der Linse zusammengestellt. Wegen der Umkehrbarkeit des Strahlenganges enthält die Tabelle mehr Information als es auf den ersten Blick scheint. Zum Beispiel stellen die Werte für $E_{2}=0,1$ und $N=0,5$ nicht nur die Daten einer Linse dar, die Elektronen von 204,4 keV auf $102,2 \mathrm{keV}$ abbremst, sondern sie beschreiben - bei Vertauschung der bildseitigen und dingseitigen Linsendaten - auch eine Linse, die Elektronen von $102,2 \mathrm{keV}$ auf $204,4 \mathrm{keV}$ beschleunigt, obwohl der letztere Wert nicht ausdrücklich als Endenergie $E_{2}$ aufgeführt ist.

Mit Hilfe der numerischen Ergebnisse und der Gln. (21) bis (23) lassen sich die Abbildungseigen-

5 W. Glaser, Handbuch d. Physik XXXIII, Springer-Verlag, Berlin 1956, S. 185. 


\begin{tabular}{|cl|c|c|c|c|c|c|c|c|c|}
\hline$N$ & & 0,1 & 0,5 & 0,8 & 2 & 4 & 6 & 8 & 10 \\
\hline$F_{\mathrm{a}}$ & $E_{2}=0,1$ & 0,6419 & 11,19 & 112,8 & 10,64 & 1,841 & 0,6329 & 0,1565 & $-0,1012$ \\
\hline$K$ & $E_{2}=1$ & 0,5641 & 11,00 & 114,9 & 11,45 & 2,054 & 0,7435 & 0,2264 & $-0,05378$ & $-0,5923$ \\
& $E_{2}=10$ & 0,4384 & 8,332 & 86,42 & 8,607 & 1,550 & 0,5430 & 0,1318 & $-0,09903$ & $-0,5650$ \\
$\Delta F_{\mathrm{a}}$ & $E_{2}=0,1$ & 0,2419 & 1,665 & 6,282 & $-2,906$ & $-1,871$ & $-1,661$ & $-1,570$ & $-1,518$ \\
\hline$K$ & $E_{2}=1$ & 0,2804 & 2,340 & 8,956 & $-3,967$ & $-2,381$ & $-2,023$ & $-1,857$ & $-1,759$ & $-1,561$ \\
& $E_{2}=10$ & 0,2326 & 2,090 & 8,350 & $-4,152$ & $-2,738$ & $-2,435$ & $-2,292$ & $-2,203$ & $-1,987$ \\
$f_{\mathrm{a}}$ & $E_{2}=0,1$ & 0,4000 & 9,525 & 106,5 & 13,54 & 3,712 & 2,294 & 1,726 & 1,416 & 0,8300 \\
\hline$K$ & $E_{2}=1$ & 0,2837 & 8,657 & 105,9 & 15,41 & $\mathbf{4 , 4 3 5}$ & 2,767 & 2,084 & 1,706 & 0,9862 \\
& $E_{2}=10$ & 0,2059 & 6,242 & 78,07 & 12,76 & 4,288 & 2,978 & 2,424 & 2,104 & 1,422 \\
$F_{\mathrm{e}}$ & $E_{2}=0,1$ & $-0,05378$ & 10,96 & 112,7 & 10,87 & 2,290 & 1,197 & 0,7970 & 0,5931 & 0,2546 \\
\hline$K$ & $E_{2}=1$ & $-0,09903$ & 10,78 & 114,8 & 11,67 & 2,497 & 1,305 & 0,8659 & 0,6419 & 0,2716 \\
& $E_{2}=10$ & $-0,2583$ & 8,110 & 86,35 & 8,825 & 1,971 & 1,075 & 0,7392 & 0,5641 & 0,2597 \\
$\Delta F_{\mathrm{e}}$ & $E_{2}=0,1$ & $-1,759$ & $-3,113$ & $-7,710$ & 1,513 & 0,4982 & 0,2971 & 0,2114 & 0,1639 & 0,07720 \\
\hline$K$ & $E_{2}=1$ & $-2,203$ & $-4,212$ & $-10,795$ & 2,232 & 0,7440 & 0,4426 & 0,3134 & 0,2419 & 0,1118 \\
& $E_{2}=10$ & $-2,231$ & $-4,087$ & $-10,35$ & 2,162 & 0,7622 & 0,4768 & 0,3516 & 0,2804 & 0,1423 \\
$f_{\mathrm{e}}$ & $E_{2}=0,1$ & 1,706 & 14,07 & 120,4 & 9,357 & 1,792 & 0,9003 & 0,5855 & 0,4292 & 0,1774 \\
\hline$K$ & $E_{2}=1$ & 2,104 & 15,00 & 125,6 & 9,439 & 1,753 & 0,8627 & 0,5525 & 0,4000 & 0,1598 \\
& $E_{2}=10$ & 1,973 & 12,20 & 96,69 & 6,663 & 1,209 & 0,5986 & 0,3876 & 0,2837 & 0,1174 \\
\hline
\end{tabular}

Tab. 1. Daten einer Rohrlinse für verschiedene Potentialverhältnisse $N=V_{2} / V_{1}$ und Endenergien. Die Werte $E_{2}=0,1,1$ und 10 entsprechen Energien von 102,2 keV, 1,022 MeV und 10,22 MeV, sofern es sich bei den beschleunigten Teilchen um Elektronen handelt.

schaften elektrostatischer Linsen in konkreten Fällen leicht überblicken.

\section{Diskussion der Ergebnisse}

Hier interessiert weniger der Verlauf der Linsendaten selbst (der qualitative Verlauf ist derselbe wie im nichtrelativistischen Fall) als die Verhältnisse von relativistischen zu nichtrelativistischen Daten. Sie sind in den Abb. 6 und 7 als Funktion des Spannungsverhältnisses $N=V_{2} / V_{1}$ an der Linse dargestellt. Parameter ist hier $E_{2}=e V_{2} / 2 m_{0} c^{2}$, die End- energie in Einheiten der Ruhmasse der beschleunigten Teilchen. Der Knick in den Kurven bei $N=1$ kommt daher, daß die Abszisse für $N<1$ um das Zehnfache gestreckt wurde. Die Energieabhängigkeit der bezogenen dingseitigen Linsendaten erkennt man am besten aus der Abb. 8 .

Vergleicht man die dargestellten Kurven mit denen von BAS und Mitarbeitern ${ }^{2 b}$, so fällt sofort die gute qualitative Übereinstimmung ins Auge (man beachte, daß das dortige $\varkappa$ unserem $1 / N$ entspricht). Quantitative Unterschiede sind natürlich vorhanden. Das muß so sein, denn unseren numerischen Werten

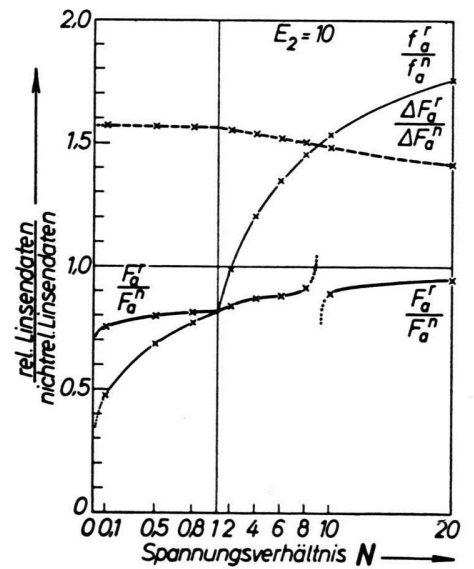

(a)

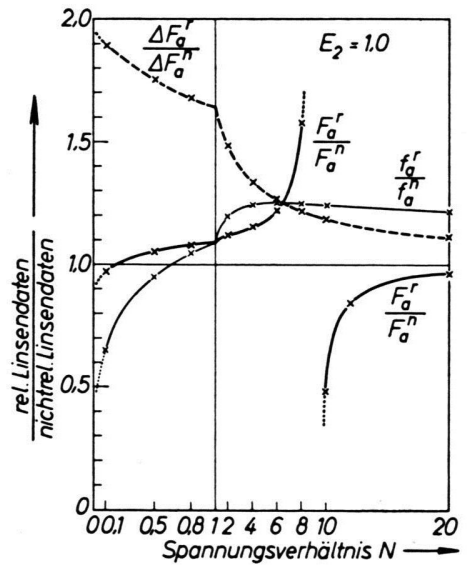

(b)

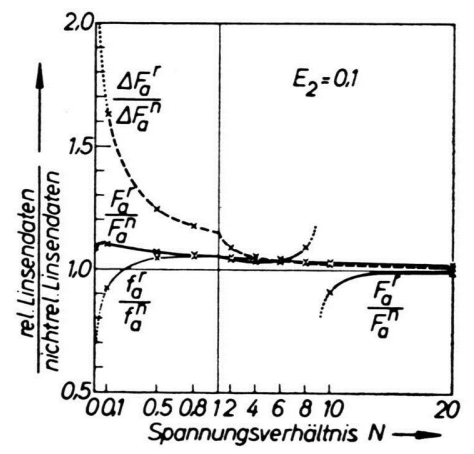

(c)

Abb. $6 \mathrm{a}-$ c. Bildseitige relativistische Linsendaten bezogen auf die entsprechenden nichtrelativistischen Daten als Funktion des Spannungsverhältnisses $N=V_{2} / V_{1}$ an der Linse. Parameter: Endenergie $E_{2}=e V_{2} / 2 m_{0} c^{2}$. Pol von $F_{\mathrm{a}} r / F_{\mathrm{a}}{ }^{n}$ bei $N=9$. 


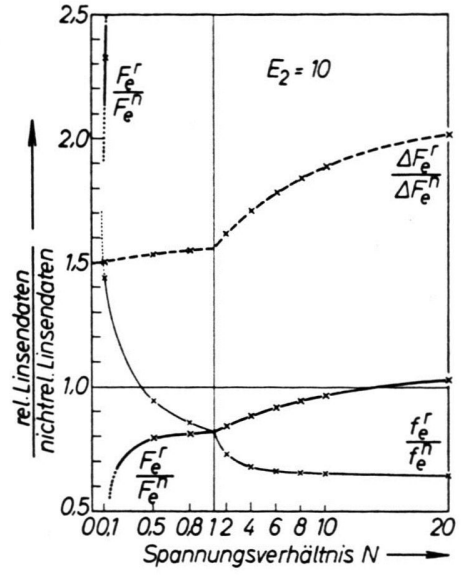

(a)

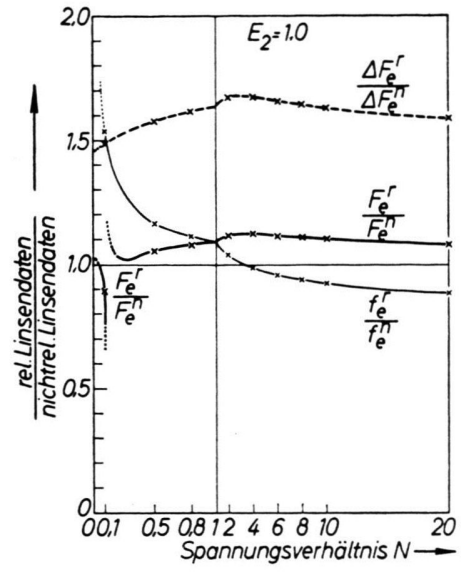

(b)

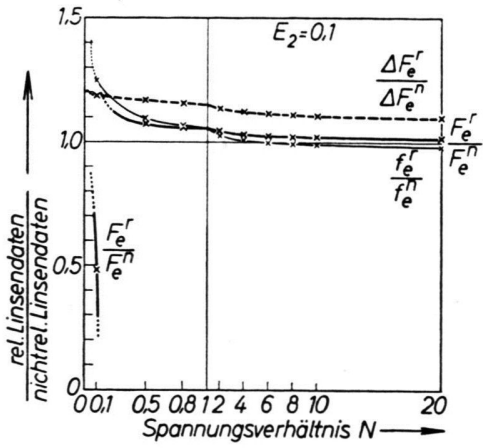

(c)

Abb. $7 \mathrm{a}-$ c. Dingseitige relativistische Linsendaten bezogen auf die entsprechenden nichtrelativistischen Daten als Funktion des Spannungsverhältnisses $N=V_{2} / V_{1}$ an der Linse. Parameter: Endenergie $E_{2}=e V_{2} / 2 m_{0} c^{2}$. Pol von $F_{\mathrm{e}}^{r} / F_{\mathrm{e}} n$ bei $N=\frac{1}{9}$.

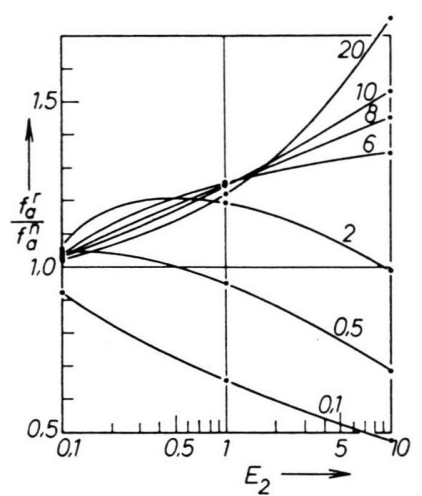

(a)

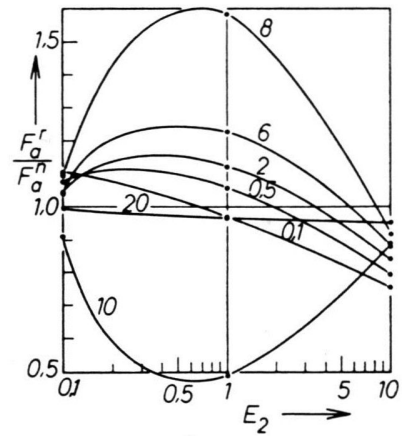

(b)

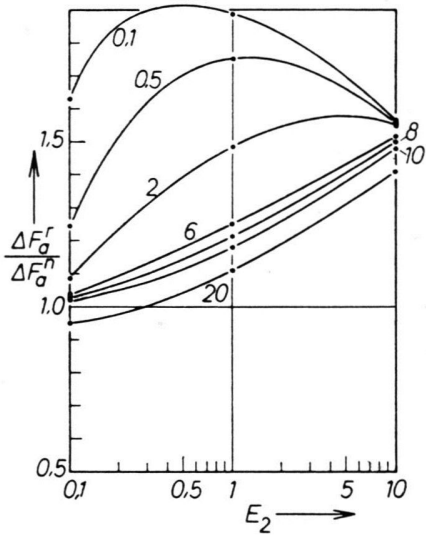

(c)

Abb. 8. Bildseitige relativistische Linsendaten bezogen auf die entsprechenden nichtrelativistischen Daten als Funktion der Endenergie $E_{2}=e V_{2} / 2 m_{0} c^{2}$. Parameter: Spannungsverhältnis $N=V_{2} / V_{1}$.

liegt ja die einfachste Approximation der Rohrlinse durch einen Streckenzug wie in A.bb. 5 zugrunde. Den Ergebnissen von BAs und Mitarbeitern liegt dagegen der genaue Potentialverlauf der von ihnen berechneten speziellen Linse zugrunde. Wenn man nicht die grobe Approximation der Abb. 5 wählt, sondern den Polygonzug genügend verfeinert, läßt sich mit den Ergebnissen des Abschnitts 2 jede Linse mit jeder gewünschten Genauigkeit berechnen. Man erhält die Abbildungsmatrix einfach durch Multiplikation von Matrizen der Formen (10) und (13).

Ein Blick auf die Kurven zeigt, daß die relativistischen Erscheinungen den Strahlverlauf im $100 \mathrm{keV}$ Bereich allmählich zu beeinflussen beginnen (diese Energieangabe bezieht sich auf Elektronen). Ihr
Einfluß nimmt mit wachsender Energie nur sehr langsam zu, so daß selbst bei einer Massenzunahme der beschleunigten Teilchen um das 20-fache $\left(E_{2}\right.$ $=10$ ) die relativistischen Linsendaten selten mehr als etwa $50 \%$ von den nichtrelativistischen abweichen. Das kommt u. a. daher, daß die Abweichungen von der Bahn des klassischen Teilchens, die durch die relativistischen Einflüsse bewirkt werden, im fokussierenden und defokussierenden Gebiet der Linse gegenläufig sind. Das erkennt man z. B. aus der Abb. 9 a, die ebenso wie die Abb. 9 b und 9 c verdeutlichen soll, wie sich die veränderten Brenndaten auf den Strahlverlauf auswirken.

Abb. 9a bzw. 9b stellt einen Fall dar, wo der Brennpunkt für relativistische Teilchen näher an der 


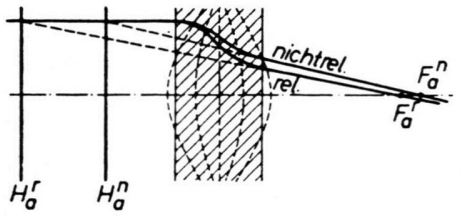

(a)

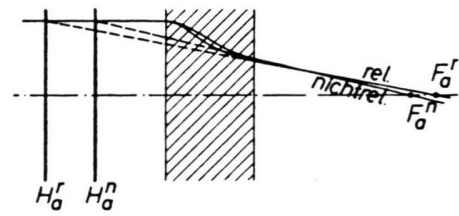

(b)

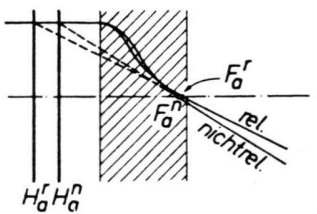

(c)

Abb. 9. Vergleich von nichtrelativistischen und relativistischen Strahlengängen; a) $\left.\left.E_{2}=10, N=4 ; \mathrm{b}\right) E_{2}=1, N=4 ; c\right) E_{2}=1$, $N=10$. Der Deutlichkeit halber wurden die Zahlenbeispiele ohne Rücksicht auf praktische Bedürfnisse gewählt und die Voraussetzung flacher, achsennaher Strahlen bei der Zeichnung nicht beachtet.

Linse bzw. weiter von ihr entfernt liegt als der für nichtrelativistische Teilchen. In beiden Fällen verläuft jedoch die Bahn des relativistischen Teilchens innerhalb der Linse näher an der Achse als die des nichtrelativistischen Teilchens. (Die Bahnkurve innerhalb der Linse läßt sich qualitativ einzeichnen, wenn man $r_{\mathrm{e}}, r_{\mathrm{e}}{ }^{\prime}, r_{\mathrm{a}}$ und $r_{\mathrm{a}}{ }^{\prime}$ [Bezeichnungen siehe Abb. 3] aus der Abbildungsmatrix ermittelt und berücksichtigt, daß die Linse in der einen Hälfte sammelnde, in der anderen Hälfte zerstreuende Wirkung hat.) Abb. $9 \mathrm{c}$ läßt erkennen, daß die stark von 1 verschiedenen Werte von $F_{\mathrm{a}}{ }^{r} / \boldsymbol{F}_{\mathrm{a}}{ }^{n}$ in der Nähe von $N=9$ keineswegs einen ungewöhnlichen Strahlenverlauf bewirken. Sie kommen nur durch den Nulldurchgang von $F_{\mathrm{a}}{ }^{n}$ bei $N=9$ zustande. Entsprechendes gilt für $F_{\mathrm{e}}{ }^{r} / F_{\mathrm{e}}{ }^{n}$ in der Umgebung von $N=1 / 9$.

Wie Abb. 9 zeigt, erfahren die relativistischen Teilchen innerhalb der Linse stärkere Ablenkungen als die nichtrelativistischen. Das kann man sich folgendermaßen leicht plausibel machen: Bekanntlich hängt die Bahn eines klassischen Teilchens - für das also der klassische Energiesatz $m v^{2} / 2=e V$ gilt - in einem elektrostatischen Feld nicht von seiner Masse ab. Die mit dem Beschleunigungsprozeß verknüpfte Massenänderung würde deshalb keine Abweichung von der klassischen Bahnkurve bewirken, wenn die Geschwindigkeit überall gerade so groß wäre, daß

$$
m v^{2} / 2=e V
$$

erfüllt wäre ( $m=$ relativistische Masse).

$$
\text { Wegen } \quad m=\left(e V+m_{0} c^{2}\right) / c^{2}=(k+1) m_{0}
$$

folgt aus (24): $\quad v=c \sqrt{2 k /(k+1)}$,

wobei $k=e V / m_{0} c^{2}$ die im Feld aufgenommene Energie der Teilchen in Einheiten der Ruhmasse ist. Gl. (26) gibt also den (hypothetischen!) Wert der Geschwindigkeit an, der trotz relativistischer Massenänderung eine klassische Bahn entstehen ließe.

Die wirkliche relativistische Geschwindigkeit berechnet sich jedoch aus

$$
\frac{m_{0} c^{2}}{\sqrt{1-\left(v_{\mathrm{rel}}^{2} / c^{2}\right)}}=e V+m_{0} c^{2}=(k+1) m_{0} c^{2}
$$

$\mathbf{z u}$

$$
v_{\text {rel }}=(c /(k+1)) \sqrt{k(k+2)} .
$$

Die wirkliche Geschwindigkeit $v_{\text {rel }}$ verhält sich also zu der Geschwindigkeit $v$, die trotz relativistischer Massenveränderung zu einer klassischen Bahn führen würde, wie

$$
v_{\mathrm{rel}} / v=\sqrt{(k+2) / 2(k+1)} .
$$

Es ist also $v_{\text {rel }} \leqq v$, wobei das Gleichheitszeichen nur im nichtrelativistischen Grenzfall gilt. Ein relativistisches Teilchen der Masse $m$ durchfliegt das Linsenfeld also zu langsam, als daß es eine klassische Bahn beschreiben könnte. Seine Bahnkrümmung ist deshalb sosowohl im sammelnden als auch im zerstreuenden Teil der Linse im allgemeinen größer als die eines nichtrelativistischen Teilchens, und zwar nimmt der Unterschied nach Gl. (29) zu, wenn das Teilchen relativistischer wird. Diese Zunahme erfolgt jedoch sehr langsam.

Die Ergebnisse der allgemeinen Rechnung lassen sich also qualitativ unmittelbar einsehen.

\section{Anwendung auf mehrstufige Beschleuniger}

Mit Hilfe der bisher dargestellten Ergebnisse lassen sich die Abbildungseigenschaften eines Systems von vielen hintereinandergeschalteten Linsen für relativistische Teilchen leicht berechnen: Die Abbildungsmatrix des Gesamtsystems ist das Produkt der einzelnen Abbildungsmatrizen. Wenn es sich um sehr viele Beschleunigungsstufen handelt, kann jedoch die Multiplikation der Matrizen recht umständlich werden.

In diesem Fall wird die Berechnung der Abbildungseigenschaften wesentlich vereinfacht, wenn man die Ergebnisse von Sonoda u. a. heranzieht ${ }^{6}$. Die Autoren ermittelten auf der Grundlage der Matrizendarstellung Rekursionsformeln, die es gestatten, die Linsendaten des aus den ersten $i$ Stufen zusammengesetzten Systems sofort hinzuschreiben, wenn die Daten des aus den ersten $(i-1)$ Stufen bestehenden Systems bekannt sind. Mit diesen Rekursionsformeln und den Gln. (20) lassen sich die elektronen-

${ }^{6}$ M. Sonoda, A. Katase, M. Seki u. Y. Wakuta, J. Phys. Soc., Jap. 15, 1680 [1960]. 
optischen Eigenschaften eines vielstufigen elektrostatischen Beschleunigers für relativistische Teilchen leicht überblicken. Sollte die den Gln. (20) zugrunde liegende 1. Näherung nicht ausreichen, läßt sich mit den allgemeinen Ergebnissen des Abschnitts 2 jede beliebige Genauigkeit erreichen, wenn man das Ach- senpotential des Beschleunigers durch einen hinreichend verfeinerten Polygonzug annähert.

Herrn Prof. Dr. W. Buckel danke ich für sein der Arbeit entgegengebrachtes Interesse und für Diskussionen. Fräulein A. Goebel danke ich für ihre sehr zuverlässige numerische Auswertung der Ergebnisse.

\title{
Die Sättigungsmagnetisierung dünner Schichten
}

\author{
Von W. DöRING * \\ Aus dem IBM-Forschungslaboratorium, Adliswil, Zürich \\ (Z. Naturforschg. 16 a, 1146--1152 [1961] ; eingegangen am 31. Juli 1961)
}

\begin{abstract}
Nach dem Heisenbergschen Modell ergibt sich für eine (100)-Schicht eines einfach-kubischen Gitters in dem Temperaturbereich $T<0,5 T_{\mathrm{c}}$, in welchem man mit ungestörter Superposition von Spinwellen rechnen darf, nur eine verhältnismäßig geringe Abnahme der Sättigungsmagnetisierung mit abnehmender Schichtdicke, nämlich bei $T=0,5 T_{\mathrm{c}}$ nur ein Absinken um $15 \%$ bei Verminderung der Schichtdicke auf 10 Atomlagen und bei tieferen Temperaturen noch weniger.
\end{abstract}

Eine Berechnung der Abhängigkeit der Sättigungsmagnetisierung dünner Schichten von der Schichtdicke wurde von KLeIN und SMIth ${ }^{1}$ für ein einfachkubisches Gitter mit einer (001)-Ebene parallel zur Schicht durchgeführt. GLAss und KLEIN ${ }^{2}$ erweiterten die Rechnung auf andere Gittertypen und andere Lagen der Schicht im Gitter. In dieser Theorie wird, ebenso wie in der Blochschen Theorie, welche zum $T^{s / 2}$-Gesetz führt, das Heisenbergsche Modell lokalisierter S-Elektronen verwendet.

Die Energie der tiefsten Zustände wird als Summe der Energien $\varepsilon(\mathfrak{f})$ unabhängiger Spinwellen geschrieben. $\varepsilon(\mathfrak{f})$ wird nach den Komponenten $k_{x}$ und $k_{y}$ des Ausbreitungsvektors $\mathfrak{f}$ parallel zur Schichtebene entwickelt, und davon wird nur der erste Summand proportional $k_{x}{ }^{2}+k_{y}{ }^{2}$ berücksichtigt. Das ist sicher nur bei Temperaturen weit unterhalb des CuriEPunktes zulässig. Dadurch wird der Vergleich der Ergebnisse mit dem Experiment sehr erschwert, denn Messungen wurden bisher nur bei Zimmertemperatur durchgeführt, und diese liegt bei den untersuchten Substanzen außerhalb des Gültigkeitsbereiches des $T^{3 / 2}$-Gesetzes.

Um einen solchen Vergleich trotzdem zu ermöglichen, haben Drigo ${ }^{3}$ einerseits und Crittenden und HofFMANN ${ }^{4}$ andererseits Verfahren zur Extrapolation der theoretischen Ergebnisse unter Benutzung

1 M. J. Klein u. R. S. Smith, Phys. Rev. 81, 378 [1951].

2 S. J. Glass u. M. J. Klein, Phys. Rev. 109, 288 [1958].

3 A. Drigo, Nuovo Cim. 8, 498 [1951]. der gemessenen Werte für die Sättigungsmagnetisierung am kompakten Material angegeben. Stichhaltige Begründungen für die Richtigkeit dieser Verfahren gibt es bisher nicht. Es besteht im Gegenteil Grund zur Annahme, daß dadurch eine zu starke Abhängigkeit der Sättigungsmagnetisierung von der Schichtdicke vorgetäuscht wird. Denn aus einer Berechnung von VAlenta ${ }^{5}$, in welcher die Austauschwechselwirkung durch ein vom Abstand von der Oberfläche abhängiges WeIsssches Feld ersetzt wird, ergibt sich ein merkliches Absinken der Sättigungsmagnetisierung unter den Wert des kompakten Materials erst bei viel geringeren Schichtdicken als nach der von Crittenden und Hoffmann modifizierten Theorie von Klein und Smith. Da die Weisssche Theorie für eine Wechselwirkung mit sehr vielen Nachbaratomen richtige Ergebnisse liefert, ist es unwahrscheinlich, daß sie für eine dünne Schicht zu qualitativ anderen Ergebnissen führt als die Spinwellentheorie.

Deshalb wurde hier versucht, die Theorie von KLeIN und Sмith auf höhere Temperaturbereiche zu erweitern. Das ist ohne Schwierigkeit möglich, weil nach Dyson ${ }^{6}$ die Wechselwirkung zwischen den Spinwellen erst bei ziemlich hohen Temperaturen eine Rolle spielt. Wenn man die Sättigungsmagnetisierung nach Potenzen von $T$ entwickelt, hängen die

4 E. C. Crittenden u. R. W. Hoffmann, Rev. Mod. Phys. 25, 310 [1953].

5 L. Valenta, Czech. J. Phys. 7, 127 [1957].

${ }^{6}$ F. J. Dyson, Phys. Rev. 102, 1217 [1956]. 